\title{
Additive manufacturing revolution in ceramic microsystems
}

\author{
Witold Nawrot and Karol Malecha \\ Faculty of Microsystem Electronics and Photonics, Wroclaw University of Science and Technology, Wroclaw, Poland
}

\begin{abstract}
Purpose - The purpose of this paper is to review possibilities of implementing ceramic additive manufacturing (AM) into electronic device production, which can enable great new possibilities.

Design/methodology/approach - A short introduction into additive techniques is included, as well as primary characterization of structuring capabilities, dielectric performance and applicability in the electronic manufacturing process.

Findings - Ceramic stereolithography (SLA) is suitable for microchannel manufacturing, even using a relatively inexpensive system. This method is suitable for implementation into the electronic manufacturing process; however, a search for better materials is desired, especially for improved dielectric parameters, lowered sintering temperature and decreased porosity.

Practical implications - Relatively inexpensive ceramic SLA, which is now available, could make ceramic electronics, currently restricted to specific applications, more available.

Originality/value - Ceramic AM is in the beginning phase of implementation in electronic technology, and only a few reports are currently available, the most significant of which is mentioned in this paper.
\end{abstract}

Keywords 3D printing, Additive manufacturing, Ceramics, Low-temperature cofired ceramics (LTCC), Photocurable resin, Stereolithography

Paper type Research paper

\section{Introduction}

Ceramics is one of the most advanced materials of our time. Whenever there are demanding conditions, such as harsh chemical environment, high thermal loads or extreme pressures, then ceramics is the material of choice. A number of electronic applications for ceramics have already been shown, using mostly low-temperature cofired ceramics (LTCC), from high-voltage (Dąbrowski et al., 2018), hightemperature (Dziedzic and Nowak, 2013) circuits and thermoelectric transducers (Markowski et al., 2019) to highly integrated devices, plasma reactors (Macioszczyk et al., 2016), microwave (Malecha et al., 2019) and microfluidic (Nawrot et al., 2018a, 2018b) structures. It has been proven many times that ceramic is a versatile material, unparalleled for complex microsystem manufacturing. Our current efforts are focused on new manufacturing techniques for highly integrated ceramic microsystems, especially those with embedded microfluidic and optical structures (Nawrot et al., 2018a, 2018b). The widely known LTCC manufacturing process is semi-additive, with subtractive layer machining and additive lamination. However, it is quite complex and hard to automate, especially if complex structures, wide channels or chambers are required. Fully additive manufacturing (AM) of ceramics could be not only a viable alternative but also a very

The current issue and full text archive of this journal is available on Emerald Insight at: https://www.emerald.com/insight/1356-5362.htm

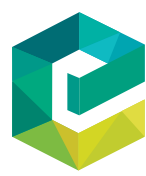

Microelectronics International

37/2 (2020) 79-85

Emerald Publishing Limited [ISSN 1356-5362]

[DOI 10.1108/MI-11-2019-0073] powerful new technology. The additive technology, in general, is already one of the most significant new technologies of our time, and it seems like just the beginning of a manufacturing revolution. The number of publications in this field has risen over five times during the past five years. Every year there are several significant innovations. There are now dozens of different processes, and the list of available materials contains more than just polymers. There are already several metal printing methods adapted in the industry (Buchanan and Gardner, 2019), and now also ceramics can be manufactured additively. The overall goal of this work is to assess the capability and applicability of ceramic AM in microsystem technology.

AM has many benefits, first of which is almost unlimited spatial structuration. In all AM processes, the part is created layer by layer, so there is virtually no need for assembly. Moreover, the material can be added in almost any place where

\footnotetext{
(C) Witold Nawrot and Karol Malecha. Published by Emerald Publishing Limited. This article is published under the Creative Commons Attribution (CC BY 4.0) licence. Anyone may reproduce, distribute, translate and create derivative works of this article (for both commercial and non-commercial purposes), subject to full attribution to the original publication and authors. The full terms of this licence may be seen at http://creativecommons.org/licences/by/4.0/legalcode
}

This research was funded by National Science Centre Poland (grant no. 2016/23/B/ST7/00932) and through statutory funds from the Wroclaw University of Science and Technology, Faculty of Microsystem Electronics and Photonics. The authors would like to thank dr hab. inż. Piotr Słobodzian and mgr inż. Krzysztof Szostak for dielectric characterization of developed structures.

Received 15 November 2019

Revised 9 January 2020

Accepted 17 February 2020 
a cutting tool would not be able to subtract. Very often AM also involves lower cost of equipment. For example, a typical desktop stereolithography (SLA) machine has a similar - often lower - price to a numerically controlled milling machine, required for LTCC technology. Furthermore, SLA does not require any additional equipment, whereas at least a press is additionally needed for LTCC, not to mention that, nowadays, lasers are mostly used instead of milling machines and have an entirely different price range. AM had been used for decades as a rapid prototyping technique because of unparalleled development speeds. Very short project-to-object time not only helps to bring products to the market faster but also is a key element for manufacturing flexibility, often a key property in the modern industry with the ever-growing demand for customization. In typical manufacturing processes, custommade tools, forms, masks, screens and, sometimes, even whole machines need to be ordered and then changed between processes. Most AM techniques, including SLA, simplify the process significantly. For the most part, there are no tools in direct contact with the object. Making changes in a manufactured product is as effortless as the modification of a computer model and transferring it to the machine. AM is also very flexible in production scaling, as there is no need for tool change. It is also easy to automate, which brings down labor costs and enhances output capabilities of the factory, which was one of the major issues in the manufacturing of complex LTCC structures. Finally, AM produces significantly less waste, as there is no material subtracting, which is better for not only cost but also the environment.

$\mathrm{AM}$ is quite a capacious term. There are many techniques (Chen et al., 2019), which can be divided into three groups: extrusion modeling, powder processing and photopolymerization. The first is the most common group and most highly popularized in recent years, especially the fused deposition modeling method, where a thermoplastic polymer is extruded layer by layer into a defined shape. It is the least expensing - in both equipment and materials - and does not require a laboratory environment. The second group is powder processing - two methods are widely popular: selective laser sintering and binder jetting. In the first case, powder particles of thermoplastic polymer, metal or ceramics are fused together using focused light. In the other case, an adequate binder glues the particles of polymer or ceramics together and then, after printing, the excessive material is cleaned off and these are fused together during a thermal process. Importantly, this method allows for multimaterial printing by using several jet nozzles containing binders with different properties, as was demonstrated with ceramics and conductive binders for electronic applications (Schulz et al., 2018). The third group of AM methods is based on the photopolymerization process. It uses resins comprising monomers and oligomers, as well as a photoinitiator. Under ultraviolet radiation, the photoinitiator generates free radicals, which in turn break the double bonds in monomers and oligomers, causing them to polymerize. This group contains SLA, where the resin is irradiated selectively, as well as multi-jet printing, where the photocurable material is deposited selectively and then a whole layer is cured with ultraviolet light simultaneously. The former is currently the base of our research. There are several SLA setups, with the main difference in the light source. The best resolution can be achieved with a laser; however, digital light projection units (DLPs) are also popular because of their increased speed of irradiation, as the whole layer is treated simultaneously. Liquid crystal displays (LCDs) are similar in that respect; however, the resolution does not match that of DLPs. On the other hand, it is the least expensive and more compact, being the solution of choice for the consumer market. In our research, we use the former SLA system, where a laser system, composed of a source and galvanometers, selectively irradiates resin through the bottom of a tank. Cured material adheres at first to a platform and then to the previous layer of the object. This object is created often at an angle, and a support structure is created to help the manufacturing process. There are very few reports on implementing ceramic SLA into the electronic manufacturing process (Nguyen et al., 2010).

\section{Materials and methods}

SLA can be used for fully ceramic part manufacturing. Currently, there are only three ceramic resins commercially available, all of them quite recent and all of them still under development. A lack of data was an incentive for characterization. There is still much to be done, even with these resins out in the market, for example, sintering profile optimization, measurement of electric and thermal properties, assessment of shape and dimension limitations in microsystems, development of bonding methods and integration into the electronic manufacturing processes.

Ceramic resins for SLA are composed of monomers and oligomers (usually of acrylates), ceramic particles and a photoinitiator [Figure 1(a)]. During the manufacturing process, resin is selectively irradiated with ultraviolet or nearly ultraviolet (most common wavelength is $405 \mathrm{~nm}$ ) light. It causes a removal of functional groups in the photoinitiator and generation of free radicals [Figure 1(b)], for example:

$$
\mathrm{NH}_{4}-\mathrm{O}-\underset{\mathrm{S}}{\|} \begin{gathered}
\mathrm{O} \\
\| \\
\mathrm{O}
\end{gathered}
$$


The free radicals generated from the photoinitiator break double bonds in monomers and oligomers, thus turning them to free radicals themselves [Figure $1(\mathrm{c})$ ], for example:

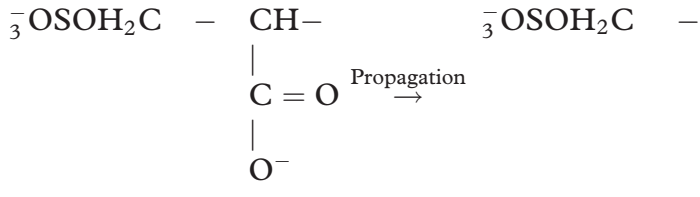

In other words, monomers become very reactive and can connect with each other, creating a polymer. Thus, ceramic particles are trapped among the polymeric structure, forming a polymer-ceramic composite [Figure 1(d)]. After the shape is defined, the polymer is burnt out [Figure 1(e)]. Differential scanning calorimetry (DSC) analysis shows that this occurs between $200^{\circ} \mathrm{C}$ and $500^{\circ} \mathrm{C}$ [Figure 2 (a)]. In the last stage, i.e. sintering, ceramic particles are fused together and the shrinkage occurs [Figure 1(f)]. In ceramics, the shrinkage is commonly anisotropic and is usually higher along the vertical axis. In ceramic SLA, the shrinkage is additionally higher in the printing direction. Shrinkage is about 15 per cent in the $x y$ plane and 25 per cent in the $z$-direction if it is the same in printing and sintering.

Figure 1 Manufacturing process of ceramic SLA

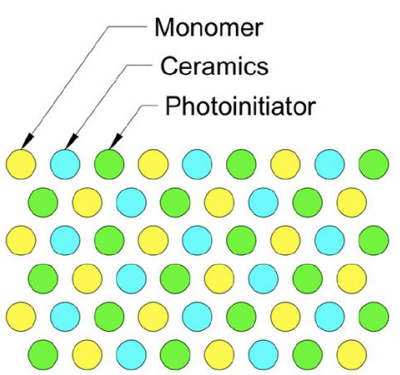

(a)

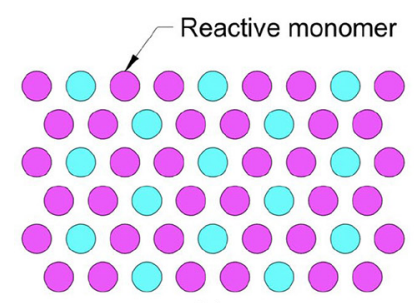

(c)

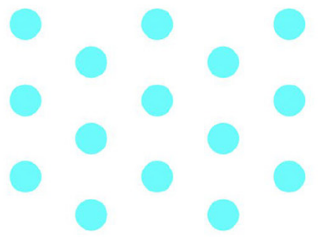

(e)

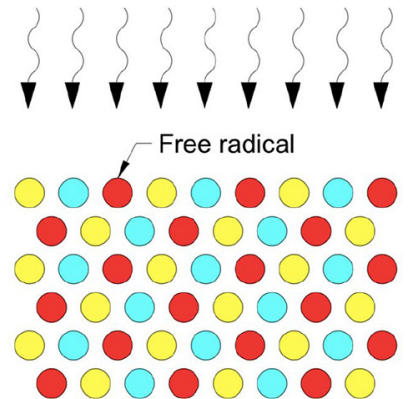

(b)

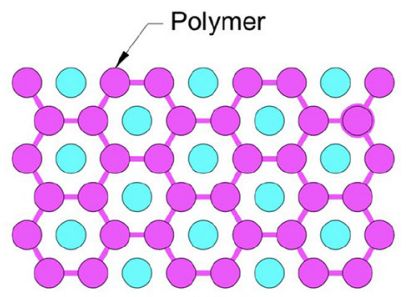

(d)

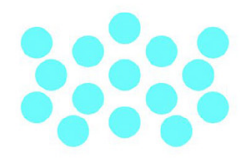

(f)
Generally, the idea of ceramic SLA resins is quite similar to LTCC, which is the most significant ceramic electronic technology. In both cases, the process starts with an unformed mixture of ceramics and necessary additives. In LTCC, these are glass to lower the sintering temperature, polymer to join the particles together in the unfired state and solvent, which enables the tape casting process, in which the mixture is formed into thin layers and then dried. Afterwards, the polymerceramic composite is shaped, mostly through cutting and milling each layer. Then, these are stacked together and laminated. The last step is sintering, where the polymer, which was binding the ceramics and glass together up to this point, is burnt out and particles are fused together. In ceramic SLA, these additives are monomers and oligomers, which form the polymer; a photoinitiator, which triggers the polymerization process; and a solvent, which provides proper rheology. Then, the polymer-ceramic composite is shaped by selective photopolymerization. Afterward, the excess of the resin is washed off and the actual part is sintered. In this process, the polymer is burnt out - as in LTCC - and the fully ceramic part is created. The overall manufacturing procedure in ceramic $\mathrm{AM}$ is as follows:

1 Preparation;

2 Build platform sanding, to obtain proper adhesion;

3 Resin stirring, to obtain uniform ceramic particle distribution;

4 Resin heating, to obtain optimal viscosity;

5 Spatial structuring using ultraviolet irradiation;

6 Washing in isopropyl alcohol (10 min);

7 Postcuring using ultraviolet irradiation (30 min);

8 Glazing (optional); and

9 Firing (schedules shown in Figure 5).

Structures presented in this paper were developed on a Formlabs Form2 3D printer using a Formlabs ceramic resin. After printing, these were rinsed in isopropyl alcohol for $10 \mathrm{~min}$ and then fired according to profiles desribed in the next section and shown in Figure 5. Investigations were carried out through DSC analyses, both with (photo-DSC) and without (DSC) light irradiation, using Mettler-Toledo DSC1, and thermogravimetric analyses (TGA), using Mettler Toledo TGA2. Dielectric characterization was carried out using split post dielectric resonator at $2.46 \mathrm{GHz}$.

\section{Results}

The TGA [Figure 2(a)] shows that this resin has about $65 \mathrm{Wt} . \%$ of ceramics. The burnout occurs between $200^{\circ} \mathrm{C}$ and $500^{\circ} \mathrm{C}$. 
Figure 2 Results of the TGA
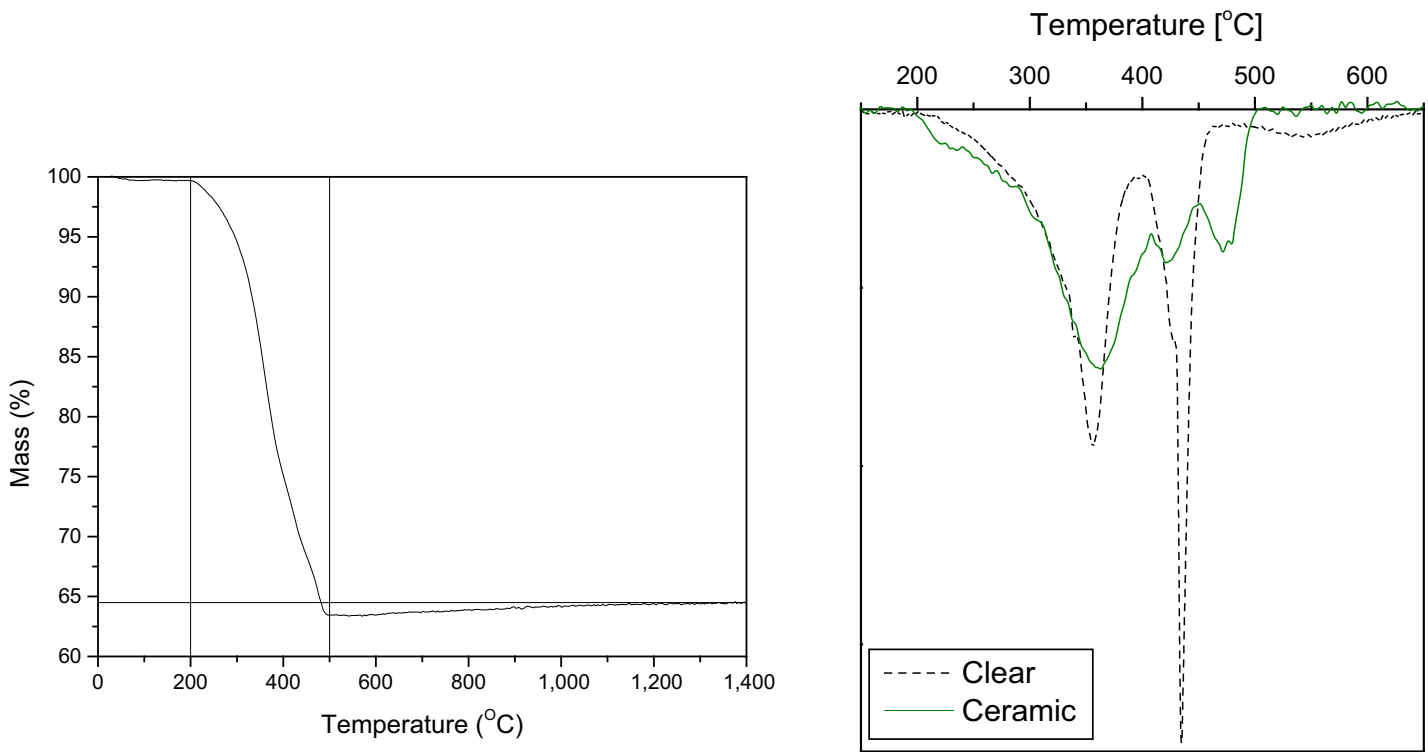

The first derivative of this curve [Figure 2(b)] shows the speed of burnout and its peaks at about $350^{\circ} \mathrm{C}, 420^{\circ} \mathrm{C}$ and $475^{\circ} \mathrm{C}$. Comparison with a fully polymeric resin from the same producer (Formlabs Clear) allows for the conclusion that these have a similar composition with an addition of a stabilizer in the case of the ceramic resin. Furthermore, it can be clearly seen that the ceramic particles restrain the polymer burnout. The rate of weight loss in the first cycle is lower and the time taken is longer in comparison with the clear resin, whereas their integrals are still comparable. The second cycle starts and ends in the same temperatures (ca. $400-450^{\circ} \mathrm{C}$ ), but the peak is lower, as there is less polymer in the ceramic resin. The third cycle is most likely as a result of stabilizer addition in the case of the ceramic resin.

To assess the applicability of ceramic SLA in microfluidics, a series of channels, with width - before firing - ranging from 500 to $1,000 \mu \mathrm{m}$, were manufactured. Owing to shrinkage, the dimensions after firing were smaller. There was no deformation during the sintering. The fidelity was very good. Therefore, another series of channels were manufactured - with smaller dimensions -to find the lower limit of structuration (Figure 3). Before sintering, their width ranged from 350 to $500 \mu \mathrm{m}$.

The cross section of the unfired structure shows that the smallest channel does not reach through the whole structure.
Furthermore, a significant skewing is also very visible, which is neither a design feature nor a machine fault. This is a correction for printing at an angle, when the vertical axis of printing will not be the vertical axis of sintering. As mentioned before, the shrinkage is anisotropic along the vertical axis of printing. This is because of the decreased concentration of ceramics in between the layers. In inverted SLA manufacturing, which was used in this research, after irradiating each layer, the structure is peeled from the bottom of the resin tank, as the irradiated resin adheres to both the printed object and the tank. In the process of peeling, some of the ceramic particles are lost from the surface. This surface then becomes an intermediate layer during the deposition of the next layer. Thus, the process causes a periodic depletion of ceramic particles, resulting in an increased shrinkage along the vertical printing axis. Therefore, a correction needs to be made. If the printing axis is not convergent with the CAM project axis, a skewing will occur. However, printing at an angle is prefered in SLA. The solution is skewing the object in the opposite direction, as a compensation. Structures were fired according to the manufacturer's profile. This process is a bit demanding, with a high peak temperature $\left(1,271^{\circ} \mathrm{C}\right)$ and a long processing time, which reaches $28 \mathrm{~h}$. The high temperature means that cofiring is

Figure 3 View of unfired series of microchannels: (a) front and (b) x-ray cross section
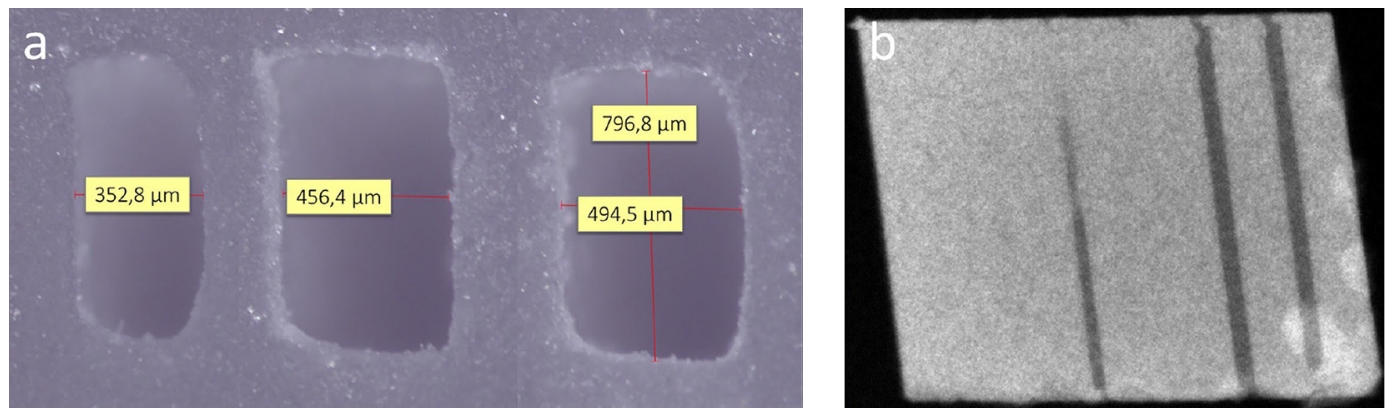
impossible with most of the thick film materials; only materials compatible with a high-temperature cofired ceramic could be used. After sintering, the smallest properly printed channel was $400 \mu \mathrm{m}$ in width [Figure 4(a)]. Therefore, shrinkage could be seen as favorable for reaching the smallest feature dimension.

The cross section of the fired structure shows [Figure 4(b)] that the correction for anisotropic shrinkage is quite accurate. Moreover, no deformation appeared during sintering. A very high porosity can also be clearly seen, which is highly undesirable for microfluidic applications, as the fluid can sink into the material. In such a case, proper rising and reusing is impossible. Therefore, a modification of the material properties is needed. A higher peak temperature was tested to induce further grain sintering. The outcome is also highly correlated with the peak hold time. An increase in either of these parameters results in a smoother and less porous structure; however, it eventually leads to deformation. The best developed high-temperature profile is shown in Figure 5. Another well-known method for surface smoothing is glazing. To reduce the number of steps, cofiring with a glaze layer was tested. In the future, glass additives to resin composition can be tested. Furthermore, shortening a firing profile with an increase in the peak temperature was tested, to see if it produces similar results. Unfortunately, such a modification results in unrepeatable results, with cracks and deformations sometimes appearing.

Dielectric properties were chosen as a measure of modification. Based on the measurements of relative permittivity and dielectric loss tangent (Figure 6), both higher sintering temperature and glaze layer addition influence material density. The shortened profile resulted in uneven surfaces, unsuitable for dielectric measurements. The additively manufactured ceramics show a relative permittivity of ca. 4 and a loss tangent of ca. $9 \times$ $10^{-3}$ at $2.46 \mathrm{GHz}$. In comparison, the typical values for the DP951 LTCC material system are a relative permittivity of ca. 8 and a loss tangent of ca. $4 \times 10^{-3}$ (Jasińska et al., 2018). Based on these results and the high porosity of developed structures, it can be assumed that the main resin component is silica. For improved electric and mechanic parameters, another ceramic, such as $\mathrm{Al}_{2} \mathrm{O}_{3}$, should be used. The possibility of thick-film deposition using screen-printing on the manufactured ceramic substrate was also investigated. For this purpose, a Du Pont $7484 \mathrm{R}$ palladium-silver paste was used. The obtained resistivity was ca. $50 \mathrm{~m} \Omega / \square$, which is significantly higher than on the alumina substrate (according to datasheet ca. $30 \mathrm{~m} \Omega / \square$ ). This is most likely because of the high porosity and roughness of the 3Dprinted ceramic substrate. Nevertheless, these parameters are acceptable and fully integrated sensor manufacturing is possible.
Figure 5 Analyzed sintering profiles

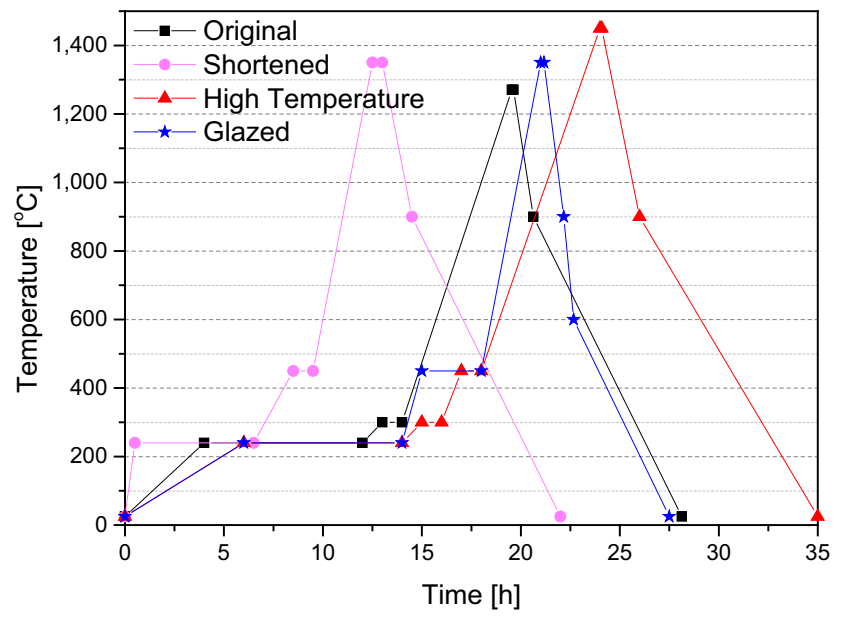

As mentioned before, the biggest issue with manufacturing channels in additively manufactured microsystems is draining the uncured resin. This can be overcome by printing the open channels and the lid separately, which provides a better access for cleaning the channels and helps to obtain smaller details. Then, the structures can be fired. Separate ceramic parts manufactured using ceramic SLA do not adhere during firing. Therefore, in the next step, a glaze layer can be screen-printed for bonding. The other layer can be deposited as well in this step, for example electrodes for electrochemical measurements. Afterward, the whole structure can be assembled and all layers cofired. The whole manufacturing process in currently available technology is shown in [Figure 7(a)], and a sample open-channel structure is shown in [Figure 7(b)]. Our further research in this area will focus on manufacturing complex electronic structures using this technology, investigating other commercially available resins and developing our own compositions.

\section{Summary}

As shown in this work, AM of ceramics has a potential to be a viable alternative for currently used LTCC. A currently available process for implementing ceramic SLA for manufacturing of even more advanced electronic structures has been shown. The described method can be used in the manufacturing of microwave and microfluidic microsystems. Ceramic material does not restrict capabilities of AM, the fidelity of printed structures is very good and firing with an

Figure 4 View of fired series of microchannels: (a) front and (b) x-ray cross section
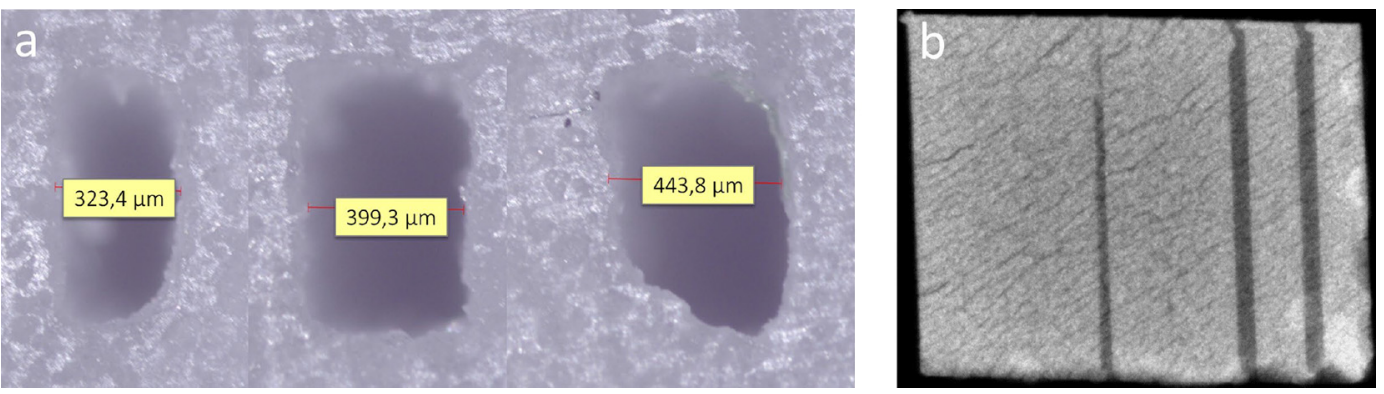
Figure 6 Results of dielectric measurements at $2.46 \mathrm{GHz}$
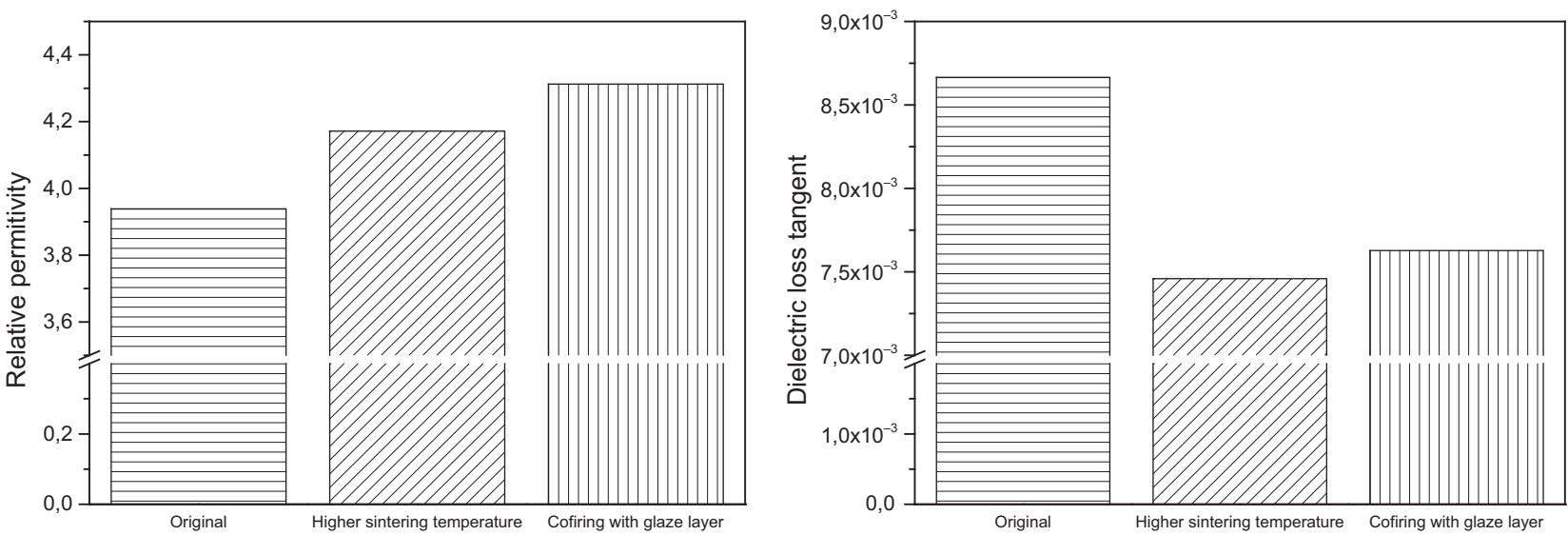

Figure 7 The manufacturing process of complex structures using open channel structures (a) and a sample structure (b)

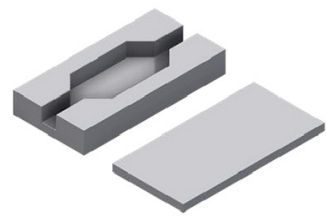

3D PRINTING FIRING

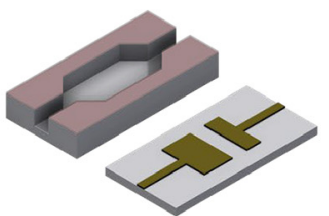

SCREEN PRINTING GLAZING

(a)

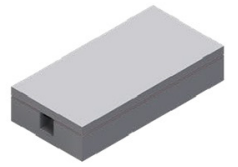

ASSEMBLING COFIRING

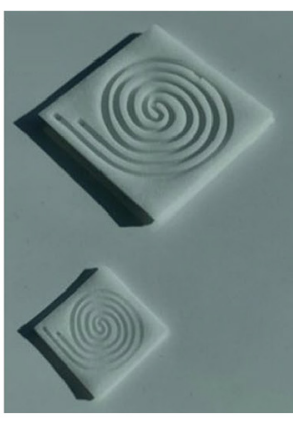

(b) optimized profile does not lead to deformation. Microfluidic channels as small as $400 \mu \mathrm{m}$ can be manufactured using this method. By using a more advanced SLA system, this parameter could be further improved. However, to unleash the true capabilities associated with AM, new materials need to be developed. These should have a decreased sintering temperature, lower dielectric losses and a less porous surface, even though a method of smoothing has been shown in this work. Still, the bulk porosity of current materials can be sometimes preferred for better thermal insulation. Our further research will focus mainly on manufacturing new compositions for improved electronic performance and optimization of the manufacturing process. Resins based on alumina seem very promising (Xu et al., 2019; Azarmi and Amiri, 2019), especially as it is a proven electronic material. Most of all, however, we are aiming to develop LTCC resins for SLA.

\section{References}

Azarmi, F. and Amiri, A. (2019), "Microstructural evolution during fabrication of alumina via laser stereolithography technique", Ceramics International, Vol. 45 No. 1, pp. 271-278.

Buchanan, C. and Gardner, L. (2019), "Metal 3D printing in construction: a review of methods, research, applications, opportunities and challenges", Engineering Structures, Vol. 180, pp. 332-348.

Chen, Z., Li, Z., Li, J., Liu, C., Lao, C., Fu, Y., Liu, C., Li, Y., Wang, P. and He, Y. (2019), " $3 \mathrm{D}$ printing of ceramics: a review", Fournal of the European Ceramic Society, Vol. 39 No. 4, pp. 661-687.

Dąbrowski, A., Rydygier, P., Czok, M. and Golonka, L. (2018), "High voltage applications of low temperature cofired ceramics", Microelectronics International, Vol. 35 No. 2, pp. 146-152.

Dziedzic, A. and Nowak, D. (2013), "Thick-film and LTCC passive components for high-temperature electronics", Radioengineering, Vol. 22 No. 1, pp. 218-226.

Jasińska, L., Macioszczyk, J., Malecha, K. and Słobodzian, P. (2018), Dielectric Characterization of Selected LTCC Materials for Microwave Applications, IEEE, Warsaw.

Macioszczyk, J., et al. (2017), "Microfluidical microwave reactor for synthesis of gold nanoparticles", Micromachines, Vol. 8 No. 11, p. 318.

Macioszczyk, J., Malecha, K. and Golonka, L.J. (2016), "The LTCC device for miniature plasma generators characterization", Microelectronics International, Vol. 33 No. 3, pp. 149-154.

Malecha, K., et al. (2019), "Monolithic microwavemicrofluidic sensors made with low temperature co-fired 
ceramic (LTCC) technology", Sensors, Vol. 19 No. 3, p. 577.

Markowski, P.M., Gierczak, M. and Dziedzic, A. (2019), "Modelling of the temperature difference sensors to control the temperature distribution in processor heat sink", Micromachines, Vol. 10 No. 9, p. 556.

Nawrot, W., et al. (2018a), "A fluorescent biosensors for detection vital body fluids' agents”, Sensors, Vol. 18 No. 8, p. 2357.

Nawrot, W., Fiedot-Toboła, M. and Malecha, K. (2018b), PDMS - LTCC Lab on Chip for Photocatalytic Effect Analysis, IEEE, Warsaw.

Nguyen, N.T., et al. (2010), "Design and characterization of $60-\mathrm{GHz}$ integrated lens antennas fabricated through ceramic stereolithography", IEEE Transactions on Antennas and Propagation, Vol. 58 No. 8, pp. 2757-2762.

Schulz, A., et al. (2018), 3D Printed Ceramic Structures Based on LTCC: Materials, Processes and Characterizations, IEEE, Warsaw.

$\mathrm{Xu}, \mathrm{X}$., et al. (2019), "Preparation of highly dispersive solid microspherical $\alpha$-Al2O 3 powder with a hydrophobic surface for stereolithography-based 3D printing technology", Ceramics International.

\section{Corresponding author}

Witold Nawrot can be contacted at: witold.nawrot@pwr. edu.pl

For instructions on how to order reprints of this article, please visit our website:

www.emeraldgrouppublishing.com/licensing/reprints.htm

Or contact us for further details: permissions@emeraldinsight.com 